\title{
Pratiques De Tableaux De Bord Dans Les PME : Une Etude Des Caracteristiques Et Raisons D'utilisation En Côte d'Ivoire
}

\author{
Tuo Katiénéfoa Seydou, \\ Doctorant en sciences de gestión, \\ Université Félix Houphouet Boigny, Côte d'Ivoire
}

Doi:10.19044/esj.2020.v16n13p111 URL:http://dx.doi.org/10.19044/esj.2020.v16n13p111

\section{Resume}

L'objectif de cet article est de comprendre les caractéristiques des tableaux de bord des PME ivoiriennes et les raisons de leur utilisation. Une étude qualitative exploratoire sur un échantillon de 21 PME révèle diverses caractéristiques et raisons d'utilisations ou non des tableaux de bord. Les résultats ont montré deux types d'entreprises. Celles dont les dirigeants affirment utiliser un TDB et celles dont les dirigeants ne l'utilisent pas à priori. Pour les premières entreprises, les tableaux de bord utilisés sont réactifs avec une fréquence de production pouvant aller du mensuel à l'année. Et un délai d'un jour en temps réel à un mois. Ils sont utilisés pour le suivi des activités, entrainer la compétition entre les employés, l'efficacité et l'obligation de résultat et la cohésion entre la hiérarchie et les opérationnels. Pour les secondes entreprises, contrairement à l'affirmation des membres des équipes dirigeantes enquêtés, le TDB existe sous forme physique en compte de résultat ou sous forme invisible dans le cognitif du dirigeant. Ils perçoivent l'outil comme réservé aux grandes entreprises, au développement de leur activité. Les pratiques informelles coexistent avec les pratiques formelles. Ce rapport serait en partie l'effet des empreintes de la culture d'oralité des peuples africains.

Mots-clés: PME, tableau de bord, contrôle de gestion, indicateur 


\title{
Dashboard Practices in SMEs: A Study of the Characteristics and Use Reasons In Cote d'Ivoire
}

\author{
Tuo Katiénéfoa Seydou, \\ Doctorant en sciences de gestión, \\ Université Félix Houphouet Boigny, Côte d'Ivoire
}

\begin{abstract}
The purpose of this article is to understand the forms that dashboards take in an SME context and the reasons for their use. A qualitative exploratory study on a sample of 21 SMEs reveals various forms and reasons for using dashboards or not. The results showed two types of businesses. Those whose leaders claim to use a dashboards and those who do not use it a priori. For the first companies, the dashboards used are reactive with a production frequency that can range from monthly to year. And from one day in real time to one month. They are used for monitoring activities, training competition between employees, efficiency and the obligation to achieve results, and cohesion between the hierarchy and operational staff. For the second companies, contrary to the assertion of the members of the surveyed management teams, this exists in physical form in income statement or in invisible form in the manager's cognitive. They perceive the tool as reserved for large companies, for the development of their activity. Informal practices coexist with formal practices. This report would be in part the effect of the imprints of the oral culture of the African peoples.
\end{abstract}

Keywords: SME, scoreboard, management control, indicator

\section{Introduction}

Les économies actuelles sont de plus en plus dominées par les petites et moyennes entreprises(PME). Elles semblent être la solution au chômage, au développement local (OCDE, 2017b, 2018). En effet, dans la zone OCDE les PME représentent la quasi-totalité de l'ensemble des entreprises. Elles sont à l'origine de $70 \%$ de l'emploi total et génèrent $50 \%$ à $60 \%$ de la valeur ajoutée en moyenne. Elles génèrent plus d'un tiers du PIB et représentent respectivement $34 \%$ et 52\% de l'emploi formel. Entre 2003 et 2016, le nombre de personnes employées à temps plein dans ces entreprises a pratiquement doublé, passant de 79 à 156 millions (OIT, 2017 p18). De ce fait les politiques économiques sont de plus en plus regardant sur ces entreprises à travers les 
reformes d'investissement, d'amélioration du climat des affaires. Dans de nombreux pays en voie de développement, les entreprises (quelles soient grandes ou petites) connaissent des difficultés qui les distinguent de leurs homologues des pays développés même si la prédominance des petites et moyennes entreprises(PME) semble mondiale. Pour être bien managé, les entreprises doivent se doter nécessairement de systèmes de décision, de planification et de contrôle contextuels (Boubakary, 2016).

La littérature sur le contrôle de gestion dans les PME est largement dominée par les études qui mettent en exergue les pratiques européennes, américaines et japonaises (Bampoky et Meyssonnier,2012). Cependant dans les pays en voie de développement, où ces entités constituent une importante source d'emplois et de renouvellement de l'économie (Julien et Marchesnay,1994), les recherches sont beaucoup plus réduites (Bampoky et Meyssonnier,2012; Torrès,1997; Ngantchou, 2016) surtout quand elles s'intéressent à un outil du contrôle de gestion. La disparition, précoce, des PME se situe entre la troisième et la cinquième année. La Côte d'Ivoire n'est pas en marge de cette hécatombe. En effet, en Côte d'Ivoire, sur dix PME crées, six disparaissent au bout de trois ans et huit au bout de cinq ans (Anassé, 2013). Pour Kouamé(2016), les causes sont liées tant à des facteurs internes qu'externes. La mauvaise organisation interne est l'une des causes impactant négativement le développement de ces entreprises. Selon les praticiens ${ }^{7}$ de l'économie ivoirienne les problèmes que rencontrent les PME ivoiriennes sont liés à la transparence dans la gestion et la qualité du management ${ }^{8}$. Ainsi les problèmes des PME ivoiriennes se trouvent liés à la qualité de leur système de gestion lui-même lié aux prises de décisions des dirigeants. Une remise en cause des outils de prise de décision utilisés par ceux-ci. En plus en contexte de pays sous-développés, l'utilisation d'outils décisionnels tel que les tableaux de bord permet d'assurer une croissance durable voire un développement dans le temps de l'entreprise (Mayegle,2010). A cet effet quels sont les outils de décisions des PME ivoiriennes ? Les tableaux de bord sont-ils mobilisés ? Quelques études (Gandaho,2007 ; Aka,2016 ; Gondo,2017) locales tentent de répondre à ces questions. Pour ces études, parmi les outils décisionnels se trouvent les tableaux de bord. Cependant elles ne révèlent aucune information sur les caractéristiques de ces tableaux de bord, ni sur les informations fournies par ceux-ci et peu sur les raisons qui poussent les dirigeants à utiliser les

\footnotetext{
${ }^{7}$ Tribune du patronat ivoirien $n^{\circ} 008$ de juillet 2014 : rencontre du mercredi 16 Avril 2014 entre le patronat ivoirien (CGECI) et la Chambre de commerce et d'Industrie ivoirienne.

${ }^{8}$ A l'issu du premier forum économique du patronat ivoirien qui s'est tenu à Abidjan du 15 au 16 Mai 2012,il est recommandé aux entreprises d' «Avoir de véritables managers qui ont une vision à moyen et long terme de leur activité et qui inspirent confiance aux banques. Avoir une comptabilité fiable, surtout les PME. Avoir recours aux agences conseils spécialisées pour l'optimisation fiscale, la structuration des transactions et l'évaluation des actifs».
} 
tableaux de bord. D'où la question centrale: comment sont les tableaux de bord des petites et moyennes entreprises? De cette question découlent les questions spécifiques suivantes: Pourquoi les dirigeants des PME utilisent les tableaux de bord? Quelles formes prennent ces outils?

L'objectif de cet article est double: connaitre les caractéristiques des tableaux de bord des PME ivoiriennes et les raisons de leur utilisation.

Outre l'introduction et la conclusion, le travail s'articulera autour de trois points. Une revue de littérature(1) permettra de faire la synthèse de la littérature tout en montrant en quoi est ce que le tableau de bord constitue un outil de pilotage des entreprises (tant grandes que petites). Ensuite nous étayerons nos choix méthodologiques(2) puis enfin les résultats(3).

\section{Revue de Litterature}

Cette partie est consacrée au cadre théorique où seront élucidés, l'utilisation des tableaux de bord comme outil de pilotage des entreprises. Un outil de pilotage des entreprises : Le tableau de bord.

\section{Tableau de bord et stratégie}

La notion de tableau de bord est différemment appréhendée dans la littérature. En effet, un tableau de bord est «un ensemble d'indicateurs choisis et conçus pour permettre au manager d'être informé de la performance passée et présente des activités qui entrent dans son champ de responsabilité, et des évènements qui peuvent influencer cette performance dans le futur» (Germain,2001). Ces indicateurs doivent être peu nombreux (cinq à dix)( Bouquin,2001) et permettre d'avoir une vue d'ensemble afin d'orienter la gestion pour atteindre les objectifs issus de la stratégie (Selmer,2003).

Pour Lorino et Tarondeau(2006), la stratégie, l'art de l'action et de la dissuasion est d'origine militaire mais peu à peu elle gagne l'entreprise à travers l'appropriation de celle-ci par les gestionnaires théoriciens et praticiens pour créer en amont les fondements de la performance. La stratégie orientée prospection influence l'équilibrage des tableaux de bord (Elhamma,2011). En effet,les tableaux de bord sont liés au deploiement de la stratégie et deux courants s'identifient : d'une part les tenants de la démarche top-down et ceux de la démarche bottom-up d'autre part. Le premier courant prône l'alignement des comportements opérationnels aux objectifs stratégiques (Kaplan et Norton,1992; 1996; 1998; 2001; Mouritsen, Larsen, Bukh et Johansen,2001). Ces tableaux de bord sont d'origine américaine et s'appuient sur le balanced scorecard. Pour le second, tableau de bord est un outil de modélisation des processus créateurs de valeur dans l'entreprise dans une logique d'apprentissage et de coordination mise en œuvre par une démarche bottomup (Epstein et Manzoni,1998; Mendoza et Zrihen,1999; Mendosa, Delmond, Giraud et Löning,2002; Wegmann, 2000, 2001; Chabin et al,2003). Cette 
démarche plus francophone concerne les tableaux de bord dits de gestion. La méthode la plus ancienne serait la méthode OVAR (Objectifs Variable d'Action Responsable).

\section{Tableau de bord : Un filtre flexible}

Benzerafa (2007), expérimente un tableau de bord balanced scorecard (BSC) dans les administrations de l'Etat français(le ministère de la défense et la caisse nationale des allocations familiales). L'outil sert à traiter un domaine périphérique au cœur du métier ou à représenter pour des fins de communication, la stratégie définie par le niveau politique. Aussi en analysant la pertinence des quatre axes définis par les auteurs du BSC pour le pilotage d'un établissement hospitalier, Nobre(2001c) ne constate-il pas que les axes processus et apprentissage organisationnel peuvent favoriser un meilleur suivi de la coordination au sein de l'hôpital et participer à l'effort d'amélioration de l'efficience. Moll, Decavel et Merlet(2009), propose comme solution au management de la qualité (l'implantation, la gestion de la qualité à travers les différentes certifications) au CHU d'Angers un tableau de bord. Dans la même l'outil est utilisé pour le suivi d'une espèce animale (Woillez et Rochard,2003). Toutefois Bourguignon et al(2002), attire l'attention sur l'importance de la contrainte culturelle dans les innovations managériales. La conception fait des outils de pilotage est parfois associée à l'automobile(Berland,2009;Fernandez,2008;Voyer,1999),l'avion(Berland,20 09;Selmer,1998), la métaphore de la marche(Bessire,2000).

Les travaux ci-dessus montrent d'une part que le tableau de bord est un outil flexible, multisectoriel dont le contenu est un ensemble d'indicateurs variables choisi afin de capter la vie passée, actuelle d'un système, d'une population organisée en vue d'anticiper en facilitant la prise de décision. Il se présente comme un filtre et permet d'assurer le dynamisme de toute organisation. D'autre part les tableaux de bord subissent l'influence de plusieurs facteurs de contingences structurelles, comportementales et culturelles. Les tableaux de bord proviennent des grandes entreprises (conception et utilisation) tout comme le contrôle de gestion. Pourtant les économies actuelles sont dominées par les petites et moyennes entreprises qui sont confrontées à des problèmes managériaux entrainant parfois des chutes prématurées(Anassé,2013; Julien,1997; Marchesnay,1991; Torrès, 1997,1998 ; Nobre,2001 ; Reyès,2004 ; Meyssonnier,2015 ; Abi Azar,2005). Qu'en est-il dans ces entreprises omniprésentes ?

\section{Tableau de bord : Outil de pilotage en contexte PME}

Plusieurs axes d'études antérieures se sont intéressés à la PME. A ce titre avant d'aborder les outils de gestion de ces entreprises; définir la PME est nécessaire. 


\section{Notion de PME}

Les définitions de la PME sont subjectives et contextuelles. Selon la commission de l'union européenne (2006 p 6), « la catégorie des PME est constituée des entreprises qui occupent moins de 250 personnes et dont le chiffre d'affaires annuel n'excède pas 50 millions d'euros ou dont le total du bilan n'excède pas 43 millions d'euros ». En Afrique, le pic lié au critère de la taille tant au niveau de l'effectif que du chiffre d'affaire est en déça. A cet effet la définition de la PME serait liée au niveau de développement. Au Cameroun «est considérée comme petite entreprise( $\mathrm{PE})$, toute entreprise qui emploie entre 6 et 20 personnes et dont le chiffre d'affaire hors taxe est compris entre 15 et 250 millions de FCFA. La moyenne entreprise (ME) emploie entre 21 et 100 personnes et dont le chiffre d'affaire hors taxe est compris entre 250 millions et 3milliards de FCFA. » (Takoudjou Nimpa et al,2018).

En Côte d'Ivoire, la loi n²014-140 du 24 mars 2014 sur les PME stipule que la petite et moyenne entreprise en abrégée PME désigne toute entreprise de production de bien et/ou service marchand qui emploi en permanence moins de deux cent personnes et qui réalise un chiffre d'affaire n'excédant pas un milliard de francs CFA. Elle est autonome, légalement constituée, tient une comptabilité régulière et s'étend à la petite et moyenne industrie en abrégé PMI. L'effectif et le chiffre d'affaire semble déterminant toutefois certaines caractéristiques liées à l'entrepreneur, sa relation avec son entreprise sont mis en évidence par les chercheurs(Guédon,2014; Marchesnay,1989; Julien et Carriere,1994; Timmons, 1994). Il est omniprésent et joue un rôle primordiale au regard de la stratégie, de la gestion, de la prise de décision et du climat organisationnel.

\section{Pilotage de la performance en contexte PME.}

La littérature sur la performance en contexte PME fait ressortir deux axes majeurs dans la mesure de la performance de ces organisations : la performance financière et la performance commerciale. Cependant d'autres axes élevés en second rang sont parfois pris en compte notamment l'innovation et le capital immatériel, l'environnement (Sogbossi,2010). La multi-dimensionnalité de la performance semble être privilégiée mais l'équilibre entre les dimensions n'est toutes fois pas établi. Plusieurs travaux sont axés sur le pilotage organisationnel par les tableaux de bord mais ceux qui s'aventurent à une étude empirique en contexte PME sont peu nombreux. Toutefois certaines études (Takoudjou et al,2018; Germain,2001,2005, 2006; Nobre,2001b) mettent en évidence des typologies et l'influence de certains facteurs de contingence sur le contenus des tableaux de bord. Ces différences de contenus des tableaux de bord dans les typologies mentionnées, reliées à des entreprises différentes, s'expliquent par le profil du dirigeant (Takoudjou Nimpa et al, 2018), la stratégie poursuivies par les dirigeants (Elhamma, 
2011). Certains travaux ( Germain,2001; Zian,2013) mettent en évidence le dégré de sophistication et l'utilisation des tableaux de bord.

\section{Parties prenantes et prises de décision}

En légitimant les intérêts des parties prenantes, la théorie des parties prenantes a contribué à faire prendre conscience aux entreprises de l'intérêt qu'elles pouvaient avoir à dépasser la simple recherche de satisfaction des actionnaires pour intégrer celle d'autres acteurs. Cela mènera finalement à un accroissement de la performance financière, et vice versa(Ulmann,1985; Clarkson,1995; Balabanis, Phillips et Lyall,1998). Les attentes de chaque acteur dépendent de la position occupée dans l'entreprise reflétée par le contrat qui le lie à l'entreprise et de certains facteurs comme le statut juridique, le contexte économique et social(Causse et Burlaud,2017). En servant ses parties prenantes l'entreprise crée peu à peu une voie de sa bonne réputation. En réalité le décideur (propriétaire et/ou dirigeant de la PME) ne cherche pas systématiquement toute l'information et ne dresse pas la liste de toutes les solutions possibles (March et Simon,1969). Ce sont les problèmes organisationnels qui sont à l'origine des décisions. Il n'existe pas d'optimum dans la prise de décision. Lorsqu'un problème se pose, le décideur utilise les processus qu'il connaît pour tenter de le résoudre (rationalité limitée).

Ces pratiques de tableaux de bord sont-elles le reflet de la représentation des pratiques des PME en Côte d'Ivoire ?

\section{Methodologie de l'etude et Recueil des Donnees Méthode et échantillonnage}

L'objectif de l'étude est de connaître les raisons d'utilisations des tableaux de bord et les caractéristiques de ces outils en contexte PME. Aussi voudrons-nous conserver une ouverture exploratoire à notre recherche. De ce fait, le paradigme épistémologique positiviste ne semble pas correspondre à notre objectif de recherche. « il génère des résultats limités en matière de contenu des perceptions » (Ayoub,2018). Quant au constructivisme, il aboutit sur des études de types recherche-action, recherche-intervention (Allard Poesi et Maréchal,2007) qui ne correspond pas à notre projet de recherche. De plus les PME sont caractérisées par l'omniprésence des dirigeants et leur influence sur leur système de gestion est connue. D'où l'expérience vécue par les dirigeants dans l'utilisation et même la conception des outils de leur système de gestion constituent un réel objectif indépendant de tout observateur. Pour la présente étude la posture épistémologique qui semble approprié est l'interprétativisme.

Par ailleur, l'approche d'étude de cas dans la recherche sur les PME offre la possibilité de mener des recherches exploratoires sur la manière dont elle est gérée dans le contexte dans lequel les processus sont appliqués 
(Romano,1989 p.35). Dans cette recherche l'étude de cas inter-site et la comparaison inter-dirigeant est privilégée pour constater des similitudes ou des différences. Pour Charreire Petit et Durieux et al(2014) 1'exploration consiste en la découverte ou l'approfondissement d'une structure ou d'un fonctionnement pour servir deux grands objectifs : la recherche de l'explication (et de la prédiction) et la recherche de la compréhension des enjeux liés à l'interaction des acteurs nécessitent des études de plus en plus qualitatives (Wacheux,1996) permettant des explications et des descriptions riches et fécondes (Miles et Huberman,2003).

Dans cette étude les PME formelles (ayant un registre de commerce et/ou des métiers) sont retenues. En plus les bases de sondage permettant d'avoir des statistiques fiables sur les PME en Côte d'Ivoire seraient limités (Aka,2016) conduisant à un choix raisonné de 21 PME. L'échantillon est constitué de 11 dirigeants,4 contrôleurs de gestion,2 comptables, 2 responsables administratifs et financiers, 1 gérante adjointe, 1 directeur technique et 1 responsable technique.

\section{Méthode de recueil et traitement des données}

Les entretiens effectués ont été enregistrés pendant une durée comprise entre 37 minutes et $1 \mathrm{H} 33$ minutes avec une moyenne d'une heure selon un guide d'entretien(en annexe). La liste du guide n'a pas pour objectif de déterminer ces enchaînements ni la formulation des questions en cours d'entretien (seul les mots clefs peuvent être repris) : l'entretien doit suivre sa dynamique propre (Combessie,2010). Ainsi le guide d'entretien est constitué d'une série de thème préalablement définis et qui peut se compléter au fil des entretiens à l'aide d'autres questions. Le fichier audio obtenu a fait l'objet d'écoute et de retranscription dans son intégralité comme le suggèrent Thiétart et al (2014 p.554) et Miles et Huberman(2003) sur «Word». Les données textuelles obtenues ont été traitées manuellement et automatiquement sous Nvivo 11 selon une analyse de contenu thématique (Moscarola,2006). Cette dernière constitue une technique de recherche pour la description objective, systématique, essentiellement qualitative et vise à interpréter un contenu (Krief et Zardet,2013).

Les données textuelles ont suivi plusieurs lectures flottantes en vue de mieux cerner le sens des propos. Les entreprises et les enquêtés ont été codifiés afin de garder leur anonymat. Deux types d'entreprises se dégagent : celles utilisant des tableaux de bord (PME 1) et celles n'utilisant pas à priori de tableau de bord (PME 2). Par ailleurs, en vue d'une triangulation des données ont été recueilli sur les caractéristiques sociodémographiques, les degrés d'utilisation et d'intégration de diverses données dans les tableaux de bord utilisés. 


\section{Resultats de l'etude}

\section{Répartition des PME enquêtées par Secteur d'activité}

Les entreprises enquêtées sont hétérogènes du point de vue de leur secteur d'activités. Un secteur domine, le secteur des services (45\%) suivi du secteur des bâtiments et travaux publics (23\%), les secteurs de la mécanique auto, du commerce, de l'agriculture(5\%) et le secteur de l'agro-industrie(5\%).

\section{Les outils utilisés dans les entreprises enquêtées}

Les propos des membres des équipes dirigeantes des PME enquêtées font ressortir plusieurs outils mobilisés par ces entreprises (Tableau 1). Elles utilisent des pratiques variées de contrôle de gestion notamment la comptabilité analytique, le budget, tableau de bord, des tableaux de suivi et des progiciels de comptabilité analytique. Les dirigeants de ces entreprises utilisent plus les outils sous forme de tableaux $(43,87 \%)$ pour le suivi des activités, de la trésorerie, de la gestion du budget $(7,55 \%)$ de leur entreprise; suivi de la comptabilité analytique $(26,88 \%)$, des progiciel $(9,91 \%)$. Et dans une moindre mesure la planification $(8,96 \%)$, le reporting $(2,93 \%)$.

Tableau 1 : Pratiques de contrôle de gestion dans les PME enquêtées

\begin{tabular}{|c|c|c|c|}
\hline Dimensions & Pratiques & $\begin{array}{l}\text { Nombre } \\
\text { de citation }\end{array}$ & $(\%)$ \\
\hline $\begin{array}{l}\text { comptabilité } \\
\text { analytique }\end{array}$ & $\begin{array}{l}\text { Soldes intermédiaires de gestion (marges sur } \\
\text { matières premières, marchandises, résultat net, } \\
\text { autofinancement, calcul de rentabilité); } \\
\text { analyse des coûts(les coûts cachés, calcul de } \\
\text { coûts du matériel, du personnel, du matériau, } \\
\text { coût de revient, coût de réalisation, coût du } \\
\text { café, réduction des coûts, des canaux de } \\
\text { distributions, éliminer les faux frais); } \\
\text { correction des écarts, taux de réalisation; } \\
\text { comptabilité analytique }\end{array}$ & 57 & 26,88 \\
\hline $\begin{array}{l}\text { tableau de } \\
\text { bord }\end{array}$ & $\begin{array}{l}\text { Tableau stratégique, tableau de bord achat, } \\
\text { tableau de bord vente, tableau de suivi des } \\
\text { taxes, tableau de suivi des chantiers; tableau de } \\
\text { suivi de la trésorerie }\end{array}$ & 93 & 43,87 \\
\hline Budget & $\begin{array}{llll}\begin{array}{l}\text { évaluation; } \\
\text { budgétaire }\end{array} & \text { suivi } & \text { budgétaire; } & \text { tableau } \\
\end{array}$ & 16 & 7,55 \\
\hline Planification & $\begin{array}{l}\text { la gestion prévisionnelle; le business plan; } \\
\text { devis et délais; plan d'action; planification des } \\
\text { activités }\end{array}$ & 19 & 8,96 \\
\hline Progiciel & $\begin{array}{l}\text { Progiciel de comptabilité analytique; veille } \\
\text { technologique et informationnelle, progiciel de } \\
\text { gestion comptable, commerciale }\end{array}$ & 21 & 9,91 \\
\hline Reporting & Reporting & 6 & 2,83 \\
\hline \multicolumn{2}{|r|}{ TOTAL } & 212 & 100 \\
\hline
\end{tabular}

Sources : auteur à partir de données sous Nvivo 11enquête 2018 


\section{Caractéristiques des tableaux de bord}

Le point précédant fait état de l'utilisation des tableaux de bord. Celuici appréhende leurs caractéristiques.

\section{Degré de sophistication des tableaux de bord}

\section{$>\quad$ Fréquence de production des tableaux de bord}

Les propos recueillis montrent à travers le tableau2 que les entreprises utilisant les tableaux de bord les diffusent annuellement, semestriellement et même mensuellement comme le souligne le contrôleur de gestion de l'entreprise BAT H : Y Y d'abord le suivi si je prends la direction technique avec laquelle je travaille beaucoup l'évaluation des chantiers se fait de façon mensuelle lorsque les chefs chantiers nous ramène les fiches de pointage que ce soit des engins et l'utilisation du matériau il ya des états qui sont sortis par rapport aux écarts (...) et ces fiches-là sont partagées avec l'administration sur support physique(sur feuille) sur lequel l'administrateur et même le directeur technique émet des commentaires. Maintenant de façon trimestrielle nous avons les réunions de sessions entre les chefs de service pour marquer un arrêt sur l'activité nous avons fait le trimestre passé à la fin du mois de juin il y a la deuxième session qui nous permet d'analyser et de tirer les conclusions ». En effet dans la même entreprise la production des tableaux de bord a lieu à des périodes différentes selon le processus d'évaluation de l'entreprise.

Par ailleurs, les tableaux de bord se diffusent également de façon hebdomadaire au sein de l'entreprise VOLACOM. "On fait notre point chaque semaine. Notre stock, notre niveau de crédit fournisseur, le bilan la caisse, les dettes par activités: le moulin, le couvoir, les fermes. On a des indicateurs. On fait cela chaque semaine.»(CG de l'entreprise VOLACOM). Cette fréquence hebdomadaire est quotidienne chez FACTORY «Ça permet d'avoir un retour journalier de tout ce qui a été fait»(DG FACTORY).

Tableau 2 : Caractéristiques des tableaux de bord

\begin{tabular}{|c|c|c|c|}
\hline Entreprises & $\begin{array}{c}\text { Production } \\
\text { de tableau } \\
\text { de bord }\end{array}$ & Fréquences de production & $\begin{array}{c}\text { Délais de } \\
\text { production }\end{array}$ \\
\hline BAT H & $\checkmark$ & $\begin{array}{c}\text { Trimestrielle; Mensuelle; } \\
\text { Quotidienne }\end{array}$ & 15 à 20 jours \\
\hline BAT B & $\checkmark$ & Semestrielle; Mensuelle & 5 jours \\
\hline CONSULT C & $\checkmark$ & Annuelle & Par mois \\
\hline PIECEX & $\checkmark$ & hebdomadaire & Un(1)jours \\
\hline GARAGE 2 & A priori non & Quotidienne & En temps réel \\
\hline FACTORY & $\checkmark$ & Quotidienne & En temps réel \\
\hline MONRIZ & $\checkmark$ & $\begin{array}{c}\text { Annuelle; Trimestrielle; } \\
\text { mensuelle. }\end{array}$ & $\begin{array}{c}\text { En temps réel; } \\
\text { Par semaine }\end{array}$ \\
\hline
\end{tabular}




\begin{tabular}{|c|c|c|c|}
\hline VOLACOM & $\checkmark$ & hebdomadaire & Une(1) semaine \\
\hline TRANSIT & $\checkmark$ & annuelle & Un(1) mois \\
\hline ALIDIST & $\checkmark$ & hebdomadaire & $\begin{array}{c}\text { En temps réel; } \\
\text { Chaque trois } \\
\text { jours au plutard }\end{array}$ \\
\hline
\end{tabular}

Source : auteur à partir de données sous Nvivo 11enquête 2018

\section{Délai de production du tableau de bord}

La réactivité des outils à travers le degré d'utilisation tel que révélée par les interviewés peut aller d'un jour en temps réel à un mois (tableau 2). En effet les propos du CG de l'entreprise ALIDIS «C'est quotidien hein on fait ça sur un certains nombre de jours mais quand ça vient net c'est le même jour on regarde.(...). On consulte chaque fois que ça revient et ça revient une fois par semaine. Et la décision se prend sur un mois. Je le consulte pendant deux heures, trois heures pour l'étudier, le comprendre et faire le récapitulatif» témoignent une production hebdomadaire, journalière et en temps réel. Celleci va de 5 jours à entre 15 et 20 jours respectivement dans les entreprises BAT $\mathrm{H}$ et BAT B.

\section{Utilisation du tableau de bord}

\section{$>\quad$ Degré d'utilisation des outils de suivi.}

Les entreprises enquêtées utilisent régulièrement les outils de suivis précis de la trésorerie $(90,9 \%)$ et des tableaux de suivi des activités (50\%). Les outils inexistants sont la mesure de performance globale (50\%), les systèmes de veille et d'informations $(45,5 \%)$, les méthodes budgétaires formalisées (45,5\%), les tableaux budgétaires $(54,5 \%)$ et les tableaux de suivis des actions des centres de responsabilité. Tandis que la mesure de la qualité du service rendu, les standards et les normes techniques sont peu utilisés $(45,5 \%)$.

\section{$>\quad$ Champ d'application}

Les propos recueillis révèlent que les entreprises enquêtées utilisent divers types de données dans leurs tableaux de bord: des données quantitatives et des données qualitatives puis des données externes. Les données quantitatives financières présentent chez les PME disposant de tableau de bord sont liées au chiffre d'affaire, la marge brute, le résultat net, au taux de recouvrement et à la trésorerie. En effet, les dirigeants de ces entreprises s'intéressent le plus au suivi de la trésorerie et de la rentabilité. «Il regarde surtout les comptes. Il regarde les comptes logés chez les fournisseurs, qu'est ce qu'ils nous doivent et qu'est-ce qu'on leur doit.»CG ALIDIST. De même que le dirigeant de l'entreprise BAT H quant à «lui ce qui l'intéresse c'est la rentabilité. Qu'est ce qu'on a gagné. Est ce qu'on est rentable, si on est perdant pourquoi est ce qu'il a perdu. L'évolution du chiffre d'affaire est un élément clé pour lui et il y a la marge dégagée par ce que nous 
on s'est donné comme à minima faire $20 \%$ sur les différents chantiers bonh» (CG de l'entreprise BAT H). L'entreprise MONRIZ intègre en plus des données financières, les données relatives aux niveaux des stocks, la quantité produite «On regarde les objectifs au niveau de la quantité en termes de chiffre d'affaire, les niveaux de stocks à atteindre à différents moments. Tout ça c'est fait en fonction de la demande. En fonction des contrats que nous avons on sait à peu près quelle quantité il faut produire par mois donc ce sont ses quantités qu'on essaie de produire pour le ramener à des objectifs par semaines.»(DG de l'entreprise MONRIZ). Outre ces données, les entreprises enquêtées utilisent également le coût de location du matériel, le délai de réalisation des travaux, des projets.

Les données qualitatives concernent la satisfaction des clients, la volonté du personnel, la qualité des matières premières et des produits, la confiance des clients et des fournisseurs, le matériel de travail et le climat social et l'environnement. En effet la «première chose quand tu viens il faut voir les enfants s'ils sont en bonne santé est ce qu'il n'y a pas quelqu'un qui est malade. Tu vois le chef de personnel si tout le monde à la santé. Ça c'est prioritaire. Deuxième priorité c'est de contrôler tout ce qui est au garage ici s'il n'y a pas quelque chose qui a manqué si tout est en place.»(DG GARAGE2). Relativement à la culture africaine, le dirigeant considère ses employés comme ses enfants. Il veille sur eux en bon père de famille. Cependant, son entreprise, le Garage n'est pas une entreprise familiale puisqu'il l'a récupéré de son défunt Directeur avec lequel il n'a aucune liaison familiale. Les données externes utilisées dans les tableaux de bord sont les données liées au coût du carburant chez l'entreprise ALIDIST, au coût de l'électricité notamment chez FACTORY. La triangulation avec les données recueillies par questions fermées à l'aide de l'échelle de Likert montrent que les données financières sont les plus élevées $(54,5 \%)$ suivies des données qualitatives $(18,2 \%)$. Les données quantitatives non financières et les données externes sont faiblement intégrées.

\section{$>\quad$ Décentralisation des tableaux de bord}

Selon le tableau 3 les tableaux de bord ne sont pas utilisés uniquement par les dirigeants des PME enquêtées. Ils s'étendent à d'autres centres de responsabilité; notamment la commercialisation, la comptabilité, la production, le service qualité, le service technique, le service achat. Cependant, les entreprises où il existe un service, une fonction contrôle de gestion; les contrôleurs de gestion rencontrés n'intègrent pas dans leurs tableaux de bord des outils en vue du suivi de leurs propres activités comme ils suivent celles des autres. 
Tableau 3 : structure des entreprises utilisant les tableaux de bord par fonction

\begin{tabular}{|c|c|c|c|c|c|c|c|c|}
\hline ENT & TECH & COM & ACH & PROD & ADM.P & SERVQ & COMP.F & CG \\
\hline BAT H & $\checkmark$ & & $\checkmark$ & & $\checkmark$ & $\checkmark$ & $\checkmark$ & $*$ \\
\hline BAT B & $\checkmark$ & & $\checkmark$ & & $\checkmark$ & & $\checkmark$ & $*$ \\
\hline $\begin{array}{l}\text { CONSULT } \\
\mathrm{C}\end{array}$ & $\checkmark$ & & & & $\checkmark$ & & $\checkmark$ & \\
\hline PIECEX & $\checkmark$ & $\checkmark$ & & & $\checkmark$ & & $\checkmark$ & \\
\hline GARAGE 2 & $\checkmark$ & & & & $\checkmark$ & & & \\
\hline FACTORY & & $\checkmark$ & & $\checkmark$ & $\checkmark$ & & $\checkmark$ & \\
\hline MONRIZ & & $\checkmark$ & $\checkmark$ & $\checkmark$ & $\checkmark$ & $\checkmark$ & $\checkmark$ & \\
\hline $\begin{array}{l}\text { VOLACO } \\
\mathrm{M}\end{array}$ & $\checkmark$ & $\checkmark$ & & & $\checkmark$ & & $\checkmark$ & $*$ \\
\hline TRANSIT & & & & & $\checkmark$ & $\checkmark$ & $\checkmark$ & \\
\hline ALIDIST & & $\checkmark$ & $\checkmark$ & & $\checkmark$ & & $\checkmark$ & $*$ \\
\hline \multicolumn{9}{|c|}{$\begin{array}{l}\text { (*)Les entreprises dans lesquelles existent une fonction contrôle de gestion } \\
\checkmark \quad \text { Oui ( utilisation de tableau de bord par le service) } \\
\text { ENT=entreprise ; TECH=technique ; COM=commercialisation ; PROD=production ; } \\
\text { ADM. P= adinistration et personnel ; COMP F.= comptable et financier ; CG=contrôle de gestion } \\
\text { ACH=achat ; SERVQ=service qualité }\end{array}$} \\
\hline
\end{tabular}

Source : auteur à partir de données d'enquête 2018

Raison d'utilisation des tableaux de bord

\section{Raison d'utilisation des tableaux de bord par les PME1}

Plusieurs raisons sont évoquées quant à l'utilisation des tableaux de bord. Ils sont utilisés pour le suivi des activités pour entrainer la compétition entre les employés, l'efficacité et l'obligation de résultat, la cohésion entre la direction et les opérationnels. Ils permettent de connaître la situation réelle en temps réelle, la maîtrise des charges (Tableau 4).

Tableau 4 : Raisons majeures d'utilisation des TDB par les PME.

\begin{tabular}{|l|l|l|}
\hline Entreprises & $\begin{array}{c}\text { Raisons } \\
\text { d'utilisation des } \\
\text { TDB }\end{array}$ & \multicolumn{1}{c|}{ Verbatims } \\
\hline BAT B & $\begin{array}{l}\text { Prise de décision } \\
\text { Orientation de la } \\
\text { stratégie et des } \\
\text { objectifs }\end{array}$ & $\begin{array}{l}\text { Chaque semestre on présente un truc général à mi- } \\
\text { annuel pour voir est ce qu'on va réorienter notre } \\
\text { stratégie ou nos objectifs(Contrôleur de gestion) }\end{array}$ \\
\hline MON RIZ & $\begin{array}{l}\text { Cohésion entre } \\
\text { hiérarchie et } \\
\text { subordonnés et } \\
\text { incitation au travail }\end{array}$ & $\begin{array}{l}\text { Au moment où je l'utilisais seul quand en } \\
\text { approvisionnement je lui criais dessus en lui disant } \\
\text { que ce n'était pas bon il ne comprenait toujours pas. } \\
\text { Mais aujourd'hui où il utilise un tableau de bord } \\
\text { avant d'arriver ici il voit et il comprend maintenant } \\
\text { mes réactions et on est tous d'accord (Dirigeant) }\end{array}$ \\
\hline FACTORY & Donne les alertes & $\begin{array}{l}\text { On sait que D.N doit faire tel tonnage par mois, le } \\
\text { jour où ça affiche moins, il ne le fait pas, comme c'est } \\
\text { un client ressource je me déplace je vais à Bouaké } \\
\text { pour aller le voir(Dirigeant) }\end{array}$ \\
\hline
\end{tabular}

Source : auteur à partir de données sous Nvivo 11enquête 2018 
Tableau de bord: des outils entre formalisation et remise en cause

Les PME 2 sont les entreprises(52,38\%) n'utilisant pas à priori de tableaux de bord de gestion. Elles ont majoritairement un chiffre d'affaire inférieure à trente millions et ayant un effectif maximum de 14 et une moyenne de 9 employés. Les membres des équipes dirigeantes ont un niveau de formation supérieur au BAC $(81,82 \%)$ (Tableau 5). Pour ces entreprises les tableaux de bord sont réservés aux grandes entreprises.

Tableau 5 : Structure des entreprises PME 2.

\begin{tabular}{|l|c|c|c|l|}
\hline ENTREPRISE & $\begin{array}{c}\text { Utilisation } \\
\text { de tableau } \\
\text { de bord }\end{array}$ & $\begin{array}{c}\text { Chiffre d'affaire } \\
\text { en millions de } \\
\text { FCFA }\end{array}$ & Effectif & \multicolumn{1}{|c|}{ Interviewé } \\
\hline CONSULT A & non & {$[0 ; 30[$} & 10 & Gérante adjointe \\
\hline MIELA & A priori non & {$[0 ; 30[$} & 4 & Dirigeant propriétaire \\
\hline ELECT & non & {$[0 ; 30[$} & 10 & Dirigeant propriétaire \\
\hline BATAGR & non & {$[0 ; 30[$} & 10 & Responsable technique \\
\hline GARAGE 1 & A priori non & {$[0 ; 30[$} & 10 & Dirigeant propriétaire \\
\hline TELEPUB & non & {$[0 ; 30[$} & 8 & $\begin{array}{l}\text { Responsable Administratif } \\
\text { et financier }\end{array}$ \\
\hline COMAG & non & {$[30 ; 150[$} & 10 & Dirigeant propriétaire \\
\hline BAT S & A priori non & {$[150 ; 1000[$} & 13 & Responsable administrative \\
\hline CONSULT S & A priori non & {$[30 ; 150[$} & 3 & Dirigeant Propriétaire \\
\hline CONSULT T & non & {$[0 ; 30[$} & 3 & Responsable Technique \\
\hline PLANTAM & non & {$[0 ; 30[$} & 14 & Dirigeant propriétaire \\
\hline
\end{tabular}

Sources : auteur à partir de données d'enquête 2018

\section{$>\quad$ Des tableaux classiques budgétaires et de suivi de trésorerie}

La gestion de ce deuxième groupe d'entreprise est axée sur l'utilisation de tableaux budgétaire. Le budget est élaboré par les différents centres de responsabilité et exécuté au niveau opérationnel après validation du cadre général y compris le budget par le directeur général comme le souligne les propos suivants : "Le tableau de bord c'est je peux dire ce n'est pas un tableau de bord nous on dit plutôt un tableau budgétaire en terme de dépenses et d'entrées d'argent. On souhaiterait par exemple sur le plan technique que vos dépenses n'excèdent pas tel montant et au point de vue commerciale vous devez réaliser tel montant de chiffre d'affaire c'est ce qu'on essaie de réaliser et les objectifs se font de façon mensuelle. Chaque fin de mois vous devez réaliser tant si vous n'avez pas atteint c'est que vous n'êtes pas arrivés mais on a une procédure interne que nous regardons (RAF TELEPUB).

Dans certaines entreprises la gestion budgétaire n'est pas utilisée. Le dirigeant utilise les tableaux de suivis de son activité et de sa trésorerie. L'échange avec l'un des responsables administratifs et financiers " j'ai un 
recap de ma situation de trésorerie. J'ai un point de mon chiffre d'affaire que je suis au fur et à mesure. J'ai un tableau de suivi, de réalisation des différents chantiers donc j'ai tel marché que j'ai commencé. Ok je suis à quel niveau voilà euhh qu'est ce qui me reste pour terminer est ce que je ne suis pas arrivé à un niveau où je peux faire un décompte solliciter un décompte pour pouvoir anticiper la fin des travaux dans peut être deux mois ou trois mois. »(RAF BAT S).

\section{Tableau de bord : une nécessité remise en cause}

Pour les membres des équipes dirigeantes des entreprises CONSULT T et COMAG, au stade de développement de leur entreprise ; la présence d'un tableau de bord n'est pas nécessaire. En effet, pour le directeur technique de CONSULT $\mathrm{T}$ son entreprise prendrait «du retard dans le traitement des problèmes des clients» s'il veut mettre en place un tableau de bord ou un manuel de procédure. Et, le directeur général de l'entreprise COMAG qualifie son entreprise de «bébé» qui aurait «besoins de bibéron» et non d'un tel outil. Quand on surcharge un bébé «on risque de l'asphyxier».

\section{$>\quad$ Des tableaux enfouis dans le cerveau.}

Les propos recueillis chez CONSULT S, GARAGE 1 et chez ELECT révèlent que les tableaux de bord existent et sont dans la «tête du dirigeant». Celui-ci s'interroge à tout moment sur la vie de son entreprise. Le dirigeant de l'entreprise GARAGE 2 affirme quant à lui utiliser un tableau de bord qui est son emploi du temps (ce qui ne correspond pas à un tableau de bord).

Les raisons de non utilisation des tableaux de bord sont dûes à divers perceptions de l'outil par les enquêtés. Il est perçu pour la plus part comme réserver aux grandes entreprises $(45,4 \%)$, un outil non nécessaire et nécessitant plus de formalisation $(18,2 \%)$ et présent de façon cognitive $(36,4 \%)$ (Tableau 6).

Tableau 6 : Raisons de non utilisation des tableaux de bord.

\begin{tabular}{|l|c|c|}
\hline \multicolumn{1}{|c|}{ Raisons } & Nombre d'entreprise & \% \\
\hline Outils réservés aux grandes entreprises & 5 & 45,4 \\
\hline $\begin{array}{l}\text { Outils non nécessaire nécessitant plus de } \\
\text { formalisation }\end{array}$ & 2 & 18,2 \\
\hline Présence cognitive & 4 & 36,4 \\
\hline \multicolumn{1}{|c|}{ Total } & 11 & 100 \\
\hline
\end{tabular}

Source : auteur à partir des données sous Nvivo 11 enquête 2018.

\section{Discussions et Conclusion}

Ce travail avait pour objectif de comprendre les formes que prennent les tableaux de bord en contexte PME et les raisons de leur utilisation. Il s'agissait de connaître les caractéristiques d'un tableau de bord en contexte PME ivoirien et les raisons qui poussent les dirigeants à les utiliser. Les 
résultats ont montré deux types d'entreprises. Celles dont les dirigeants affirment utiliser un TDB et celles qui ne l'utilisent pas à priori. Pour les premières entreprises, les propos recueillis montrent la présence de données tel que le chiffre d'affaire, la marge brute, le résultat net, le taux de recouvrement, la trésorerie, la rentabilité, la satisfaction et la confiance clientèle, le niveau des stocks, la capacité de production, la volonté du personnel, la qualité des matières premières, le climat social et l'environnement. Les données financières sont les plus élevées suivies des données qualitatives alors que les données quantitatives non financières et les données externes sont faiblement intégrées. Ils sont de plus en plus décentralisés selon l'étendue de l'activité et s'étendent à d'autres services notamment la comptabilité, la commercialisation, la production, le service technique et le service qualité. Cette diversité des données produites par les entreprises ivoiriennes corroborent les travaux de Ngongang(2005,2013), Chappellier et Hamadi(2012), Lavigne(1996). Les activités les plus courantes dans les services comptables des PME camerounaises de Douala et Yaoundé sont la comptabilité générale(92,6\%) et la fiscalité(88,2\%) (Ngongang,2013) et ces entreprises recours à l'outil informatique pour l'établissement des situations intermédiaires et le traitement de l'information comptable (Ngongang,2005).

Pour les secondes entreprises, le TDB existe sous forme physique en compte de résultat ou sous forme invisible dans le cognitif des dirigeants. Ils perçoivent l'outil comme réservé aux grandes entreprises. De ce fait, lié à la taille, au développement de l'activité de l'entreprise à travers sa formalisation. L'utilisation d'un tableau de bord est en partie liée à la volonté du dirigeant, sa perception de l'outil. Aussi cet usage serait-il lié à l'utilité de la théorie du cycle de vie en contexte PME avec les travaux de Condor(2012).

Les entreprises enquêtées mobilisent divers outils de contrôle de gestion: les tableaux de bord, le budget, la comptabilité analytique, les tableaux de suivi comme mis en évidence par les travaux de certains auteurs( Wade et Dieng,2019; Gondo,2017; Aka,2016; Nobre,2001b; Germain,2001, 2004, 2005, 2006; Zian,2013). Et l'existence de fonction contrôle de gestion dans certaines PME. Cette présence est une volonté pour les dirigeants de suivre leur activité et de formaliser leur système de gestion. Ce qui réduit en partie l'activité des contrôleurs de gestion au suivi et corroborent ainsi les travaux de Julien(1994) qui montrent une vision restreinte du contrôle de gestion par la gestion des opérations. Les pratiques budgétaires coexistent avec les tableaux de bord dans les PME sénégalaises mais celles-ci accordent une place prédominante au budget (Wade et Dieng, 2019). Par ailleurs nos travaux relativisent ceux de Abi-Azar(2005) quant à l'invisibilité de la fonction contrôle de gestion en contexte PME; et de Ngongang(2013) pour qui les outils du contrôle de gestion jouissant d'une importance élévée en contexte 
PME camerounaises sont le budget et la comptabilité analytique. Nos résultats présentent des similitudes aux résultats des travaux de plusieurs auteurs(Germain,2001 ; Zian, 2013 ; Sogbossi,2010) montrant l'utilisation d'outils réactifs et décentralisés couvrant plusieurs dimensions de la performance avec priorité accordé à la performance financière et la satisfaction clientèle puis une influence positive forte de la taille, de l'industrialisation de l'entreprise. Plusieurs raisons sont évoquées dans l'utilisation des tableaux de bord. Ils sont utilisés pour le suivi des activités, pour entrainer la compétition entre les employés, l'efficacité et l'obligation de résultat et la cohésion entre la hiérarchie et les opérationnels. Ils permettent de connaître la situation réelle en temps réel, la maîtrise des charges.

A la lumière de ces constats exploratoires, il convient de relativiser l'affirmation selon laquelle les entreprises africaines se basent que sur le suivi des informations informelles pour prendre leurs décisions de gestion (Baidari,2005). Cependant, fort est de constater que les pratiques informelles coexistent avec les pratiques formelles. Ce fait résulte en partie des empreintes de la tradition africaine axée sur la communication orale (Dia,1999). De plus les travaux sur le contrôle de gestion en contexte PME sont récents et mettent en œuvre l'introduction de celui-ci, son design, son rapport avec les théories du cycle de vie, la théorie de la contingence, la mobilisation des outils, des pratiques de contrôle de gestion. Ces études mettent en évidence une vue d'ensemble sur les pratiques de contrôle de gestion de ces entreprises sans intégrer la boite que constitue un outil et une pratique, de son élaboration, les raisons de son utilisation, et le comment de son utilisation. Dans le contexte ivoirien présentant peu de travaux sur cet angle, ce travail contribue à combler ce déficit, en s'intéressant à un outil particulier le tableau de bord. A cet effet, il envisage d'une manière différente l'introduction du contrôle de gestion dans les PME, l'élaboration d'un tableau de bord PME, les conditions de réussite de son implantation. Toutefois, cette étude n'est pas exempte de critique. La taille de l'échantillon et l'aspect qualitatif de la recherche pose un problème de généralisation des résultats même si cela n'était pas lié à l'objectif de la présente recherche.

D'éventuelles recherches sur des échantillons plus larges statistiquement significatifs pourraient mettre en évidences d'autres aspects des tableaux de bord (élaboration et rôles des acteurs) puis dans d'autres contextes. Eu égard aux différentes formes des tableaux de bord identifiées l'on pourrait s'intéresser au lien entre facteurs de contingence, les formes des tableaux de bord et le choix des modes d'organisation des PME africaines. 


\section{References:}

1. Abi-Azar (2005). les outils de contrôle de gestion dans le contexte des pme : cas des PMI au Liban. Comptabilité et Connaissances, France HAL 0581105.

2. Aka M. A. (2016). objectifs et contrôle de gestion dans la PME ivoirienne : analyse des mécanismes à partir des outils utilisés Revue internationale de gestion et d'économie série A-gestion $\mathrm{n}^{\circ} 1$ vol 1 décembre pp76-94.

3. Anassé A. (2013). Arbitrage homogénéité/taille des segments : proposition d'une approche pour les PME africaines. Revue Management international p. 124

4. Allard Poesi F., Marechal G. (2007). Construction de l'objet de la recherche, in Thiétart $\mathrm{R}$. A. et al. (Éd.), Méthodes de recherche en management, Dunod 3e Édition P. 34-57

5. Ayoub M. (2018). Configuration des outils du control package selon la théorie $d u$ couplage lâche: étude multicas des grandes coentreprises françaises basées au Qatar. Thèse executive doctorate in business administration de l'université Paris-Dauphine et de l'université Saint-Joseph de Beyrouth.

6. Baidari B. (2005). Les entreprises sénégalaises utilisent-elles les données comptables qu'elles produisent Revue Africaine de Gestion $\mathrm{n}^{\circ} 2$ Mai. $^{2}$

7. Balabanis G, Phillips, H, C, Lyallj (1998). Corporate Social Responsibility and Economic Performance in the top British Companies: are they Linked? European Business Review. 98(1) p. 2544.

8. Bampoky B. \& Meyssonnier F. (2012). L'instrumentation du contrôle de gestion dans les entreprises au Sénégal comptabilité et innovation HAL 00690927.

9. Benzerafa M. (2007). L'universalité d'un outil de gestion en question - Cas de la Balanced Scorecard dans les administrations de l'Etat thèse de doctorat Université Paris X Nanterre.

10. Berland N. (2009). Mesurer et piloter la performance e-book www.Management free.fr

11. Bessire D. (2000). Du tableau de bord au pilotage : l'entreprise au risque de se perdre. $21^{\text {ème }}$ congrès de l'AFC.

12. Boubakary (2016). Influences de facteurs de contingence sur le management des entreprises africaines : Le cas du Cameroun. Revue africaine de management vol 1 PP.133-148.

13. Bouquin(2001). Contrôle de gestion Presse universitaires de France.

14. Bourguignon A. Norreklit H.(2002). L'irréductible dimension culturelle des instruments de gestion: l'exemple du tableau de bord et 
du balanced scorecard Comptabilité- contrôle-Audit /numéro spécial mai pp.7-60

15. Causse G. \& Burlaud A. (2017). La comptabilité : universalisme ou contingence ? 2ème Journées d'Etude Africaine en Comptabilité et Contrôle(JEACC).

16. Chabin Y. Naro G. \& Travaille D. (2003). Les tableaux de bord stratégiques entre conception et action : propos d'étape d'une recherche intervention. Actes du 24ème Congrès de l'Association Francophone de Comptabilité Louvain Belgique.

17. Chapellier P. \& Hamadi Z. B. (2012). Le système de données comptables des dirigeants de PME tunisiennes : complexité et déterminants. Management international 164 (2012): 151-167.

18. Charreire-Petit S. \& Durieux F. (2014). Explorer et tester : les deux voies de la recherche in Thietart R. A. (2014). Méthode de recherche en management DUNOD PARIS.

19. Clarkson M. B. E. (1995). A stakeholder framework for analyzing and evaluating corporate social performance. Academy of Management Review, Vol. $20 \mathrm{n}^{\circ} 1$ pp. 92-117.

20. Combessi J. C. (2010). La méthode en sociologie. La découverte.

21. Condor R. (2012). Le contrôle de gestion dans les PME : une approche par la taille et le cycle de vie. Revue internationale PME. Vol $25 \mathrm{n}^{\circ} 2$ pp 77-97.

22. Dia A L.(1991). Le management africain Mythe ou réalité Revue internationale PME : Économie et gestion de la petite et moyenne entreprise. Vol. 4 n ${ }^{\circ}$ p. 29 - 48.

23. Elhamma A. (2011). Impact de la taille sur le contenu des tableaux de bord dans les entreprises au Maroc : résultats d'une étude empirique. halshs.archives-ouvertes. fr:halshs- 00670474.

24. Epstein M.J \& Manzoni J.-F(1998). Performance Measurement Management Contrôle: Superior Organizational Performance. Elsevier, Collection: Studies in Managerial and Financial Accounting Vol. 14 pp. 185-202

25. Fernandez A. (2008). Les nouveaux tableaux de bord des managers : le projet décisionnel dans sa totalité. Éditions d'Organisation Quatrième édition. Paris

26. Gandaho(2007). Pratiques et méthodes budgétaires des entreprises en Côte d'Ivoire. Revue du CAMES-Nouvelle série B Vol $008 \mathrm{~N}^{\circ} 1$ 2007(1er Semestre).

27. Germain C. (2001). Le pilotage de la performance dans les petites et moyennes entreprises les résultats d'une recherche empirique. 22ème Congrès de l'AFC Mai. 
28. Germain C. (2005). The impact of size environment structure and technology on performance measurement systems (PMSs) design: an empirical study of French small and medium-sized enterprises (SMEs) XXVIIIe Congrès de l'European Accounting Association Göteborg, 1821 mai.

29. Germain C. (2006). Le pilotage de la performance dans les PME en France : Une comparaison des pratiques de tableaux de bord des organisations familiale et des filiale. Revue internationale $P M E$ : économie et gestion de la petite et moyenne entreprise vol $19 \mathrm{n}^{\circ} 1 \mathrm{pp}$ 69-94.

30. Gondo T. C. (2017). Le contrôle de gestion en contexte pme ivoirienne : l'information comptable est elle mobilisee ? 2ème journée d'étude africaine en comptabilité et contrôle (JEACC) 15 Décembre 2017 UCAD - Dakar.

31. Guedon J. L. (2014). Le rôle des dirigeants en matière de responsabilité sociale des entreprises: une approche multicas de PME Françaises. Thèse de Doctorat Université de Sherbrooke

32. Julien P. A. \& Marchesnay M. (1992). Des procédures aux processus stratégiques dans les PME in Perspectives en Management stratégique Tome 1, pp 97-129 Editions Economica-Gestion 431 p.

33. Julien P.-A. (1997). Pour une définition des PME in Les PME : Bilan et perspectives Economica pp. 1-43.

34. Julien P.-A. \& Carriere B. (1994). L'efficacité des PME et les nouvelles technologies. Revue d'économie industrielle Vol. 67, $\mathrm{N}^{\circ} 1$, pp. 120-134.

35. Kaplan R.S. \& Norton D.P. (1992). The Balanced Scorecard - Measure that drive performance. Harvard Business Review February pp. 71-79.

36. Kaplan R. Norton D.(1996). The balanced scoredcard: translating strategy into Action. Havard Business school press: Boston. Traduction française: Le tableau de bord prospectif les éditions d'organisation 1998.

37. Kaplan R.S. \& Norton D.P. (1998). Le tableau de bord prospectif, Editions des Organisations, traduit de: The Balanced Scorecard (1996) Harvard Business School Press.

38. Kaplan R.S. \& Norton D.P. (2001). The strategy focused organisation. How balanced scorecard companies thrive in the new business environment. Harvard Business School Press USA

39. Kouame K. M. (2016). La défaillance des pme : essai de détermination d'une trajectoire. Revue internationale de Gestion et d'économie serie A-Gestion/n 1 vol 1 décembre PP 139-168. 
40. Krief N. \& Zardet V (2013). Analyse de données qualitatives et recherche- intervention. Recherches en Sciences de Gestion 2013/2 N ${ }^{\circ}$ 95 | pages 211 à 237.

41. Lavigne B. (1996). Les États Financiers des PME sont-ils seulement utiles pour des fins fiscales? Actes du XVIIème Congrès de l'AFC Université de Valenciennes Vol.2 pp. 951-966.

42. Lorino P. \& Tarondeau J-C. (2006). De la stratégie aux processus stratégiques. Revue Française de Gestion; Jan 2006; 32160 ABI/INFORM Global pg. 307

43. Marchesnay M. (1989). La petite entreprise espace de transactions Entrepreneurship and regional development de juillet 1990.

44. Marchesnay M. (1991). La PME : une gestion spécifique?, Economie Rurale, $\mathrm{n}^{\circ} 206$ pp.11 17

45. Mayegle F-X. (2010). La contribution du tableau de bord prospectif à l'amélioration des performances des entreprises : une étude à partir de six cas au Caméroun.Thése de doctorat Université de Yaoundé.

46. Mendoza C. \& Zrihen R. (1999). Le tableau de bord : en V.O. ou en version américaine ? Comparaison entre tableau de bord et le balanced scorecard », Revue Française de Comptabilité N 309 Mars pp. 60-66.

47. Mendoza C., Delmond M.H., Giraud F, Löning H. (2002). Tableaux de bord et balanced scorecard Guide de Gestion. Revue Fiduciaire.

48. Meyssonnier F. (2015). Quel contrôle de gestion pour les startups ? hal-01116382

49. Miles \& Huberman (2003). Analyse des données qualitatives. 2ème édition deboeck

50. Moll M. C. Decavel F. Merlet C. (2009). Tableau de bord d'évaluation du système qualité des pôles en établissement de santé : un outil pédagogique. Recherche en soins infirmiers 2009/3( $\left.{ }^{\circ} 98\right)$ page 19-27.

51. Moscarola J. (2006). Le Sphinx Développement, Analyse lexicale avec Le Sphinx Manuel d'utilisation $155 \mathrm{p}$.

52. Mouritsen, J., Larsen, H., Bukh, P.N. et Johansen, M. (2001). Reading an Intellectual Capital Statement: Describing and prescribing knowledge management strategies. Journal of Intellectual Capital 2(4):359-383.

53. Ngantchou A. (2016). Le contrôle de gestion en contexte PME africain : l'informalité est-elle a règle ? Communication au congrès de l'AFC Toulouse.

54. Ngongang, D. (2005).Pratiques comptables, système d'information et performance des PME camerounaises. La Revue des Sciences de Gestion Direction et Gestion 216: 59-70. 
55. Ngongang D. (2013). Système d'information comptable et contrôle de gestion dans les entreprises camerounaises. La Revue Gestion et Organisation 5 PP.113-120

56. Nobre T. (2001 a). Le contrôleur de gestion de la PME. Comptabilité Contrôle Audit Vol 1 Tome 7 PP 129-146

57. Nobre T. (2001 b). Méthodes et Outils du contrôle de gestion dans les PME. Finance Contrôle Stratégie vol 4 n² pp119-148.

58. Nobre T (2001 c). Management hospitalier : du contrôle externe au pilotage apport et adaptabilité du tableau de bord prospectif. comptabilité - contrôle - audit vol 2 (tome 7) pages 125 à 146

59. OCDE(2018). Renforcement des PME et de l'entrepreneuriat au service de la productivité et de la croissance inclusive. https://www.oecd.org/cfe/smes/ministerial/documents/2018-

Conference-Ministerielle-PME-Session-Themes-Principaux.pdf

60. OCDE (2017b). Panorama de l'entrepreneuriat 2017. Éditions OCDE Paris http: //dx.doi.org/10.1787/2017-fr. OCDE

61. OIT (2017). Emploi et questions sociales dans le monde 2017 : Entreprises et emplois durables : des entreprises formelles et un travail décent. Bureau international du travail Genève.

62. Reyes G. (2004). La moyenne entreprise est-elle spécifique ? Actes $d u$ VIIe CIFEPME, octobre Montpellier.

63. Romano, C. (1989). Research strategies for small business: A case study approach. International small business journal 7 (4): 35-43.

64. Selmer C.(1998). Concevoir le tableau de bord Paris Dunod 4è édition.

65. Selmer C. (2003). Concevoir le tableau de bord outils de contrôle, de pilotage et d'aide à la décision. Paris Dunod 2è édition.

66. Sogbossi B. B. (2010). Perception de la notion de performance par les dirigeants de petites entreprises en Afrique. La revue des Sciences de Gestion vol 1 n²41 p. 117-124.

67. Takoudjou-Nimpa A Kanga-Wendji C Tajeute-Temena K(2018). Le choix des indicateurs de mesure de la performance dans les tableaux de bord: quels liens avec le profil du dirigeant de la PME ? $3^{\text {ème }}$ Journée d'étude africaine en comptabilité et contrôle.

68. Timmons J. A.(1994). New Venture Creation: entrepreneurship for the 21st century. Chicago Irwin.

69. Thietart R. A. (2014). Fondement épistémologique de la recherché "méthode de recherche en management DUNOD PARIS

70. Torres O. (1997). Pour une contingente de la spécificité de la PME. Revue internationale PME, vol 10, n², p. 9-43.

71. Torres O. (1998). Vingt-cinq ans de recherche en gestion de la PME: une discipline entre courants et contre-courant chapitre 1 dans PME: De nouvelles approches. Editions Economica, octobre 1998. 
72. Ullmann A. (1985). Data in search of a theory: a critical examination of the relationship among social performance social disclosure, and economic performance. Academy of Management Review Vol. 10 p. 540-577.

73. Voyer P. (1999). Tableaux de bord de gestion et indicateurs de performance. (2e édition) Les Presses de l'Université du Québec

74. Wacheux F. (1996). Méthodes qualitatives et recherche en gestion. Economica

75. Wade M. E. B. et Dieng O. T. (2019). Les outils de contrôle de gestion en contexte PME au Sénégal: Une analyse de l'effet taille. Revue africaine de management VOL.4 (3) 2019 (PP.36-57)

76. Wegman G. (2000). Les tableaux de bord stratégiques: Analyse comparative d'un modèle nord-américain et d'un modèle suédois. Gestion 2000 Recherches et Publications en Management A.B.S.L.

77. Wegman G. (2001). Les tableaux de bord stratégiques : une instrumentation $\mathrm{du}$ controle de gestion stratégique concepts instrumentation et enquête. 22ème congrès de l'AFC May 2001

78. Woillez M. et Rochard E. (2003). Tableau de bord, un outil pour le suivi des poissons migrateurs : concept et réflexion sur le choix d'indicateurs pour la population de grande alose du bassin versant Gironde Garonne-Dordogne. Ingénieries - E A T IRSTEA édition 2003 p. 43 - p. 59

79. Zian H. (2013). Contribution à l'étude des tableaux de bord dans l'aide à la décision des PME en quête de performances. Thèse de doctorat en Sciences de gestion Université Montesquieu.

Annexe: Guide d'entretien (après présentation interviewer interviewé et son organisation). Comment faites-vous pour déceler les problèmes et certaines anomalies au sein de votre entreprise? Sur quelle base se fondent vos décisions ? Pour vous qu'est-ce la performance ? Quels sont les outils de gestion que vous utilisez dans la gestion de votre entreprise ? Pour vous qu'estce qu'un tableau de bord ? Utilisez-vous un tableau de bord ? Pourquoi... ? Que pouvez-vous nous dire des caractéristiques du tableau de bord que vous utilisez ? Au terme de notre entretien nous vous demandons votre mot de fin en d'autres termes que devons-nous retenir du pilotage de votre entreprise (interviewers dirigeants) et de votre centre de responsabilité (interviewers non dirigeants). 